\title{
IMPORTANCIA RELATIVA DE ESPECIES CUYO POLEN ES UTILIZADO POR APIS MELLIFERA EN EL ÁREA DE LA RESERVA NACIONAL LOS RUILES, VII REGIÓN DE CHILE
}

\author{
Gloria MONTENEGRO, Miguel GÓMEZ y Guacolda ÁVILA
}

\begin{abstract}
RESUMEN. En el presente trabajo, se ha determinado el uso selectivo que Apis mellifera hace del recurso vegetal de las comunidades de la región mediterránea sub-húmeda de Chile en su recolección de polen. Mediante muestreo sistemático de cúmulos polínicos del polen corbicular, se ha diagnosticado y cuantificado las especies preferidas por las abejas melíferas, y se ha determinado la importancia relativa de cada una de ellas.
\end{abstract}

Palabras clave. Polen corbicular, biomasa, selectividad, Chile.

ABSTRACT. In this work, we have determined the selective use of plant resource for pollen collection by Apis mellifera in the sub-humed mediterranean zone of Chile. Throughout sampling pollen loud trapped at the entrance of the hives, was quantified the different plant used species by honey bees, analyzing the relative importance of them.

Key words. Corbicular pollen, biomass, selectivity, Chile.

\section{INTRODUCCIÓN}

El interés por el conocimiento de la flora melífera de una región y su incidencia en la actividad de Apis mellifera, crece paralelamente a la importancia que han ido adquiriendo los productos resultantes de dicha actividad como miel, propóleo, jalea real y cera. Por tal razón resulta indispensable conocer también la fuente alimenticia de estas abejas, es decir, la flora polínica que permite la actividad sostenida de la colmena.

Se sabe que el polen es la fuente primaria de proteínas, lípidos, vitaminas y minerales para las abejas y cuando se mezcla con agua y miel, provee los elementos nutritivos para la producción de secreciones glandulares indispensables para el crecimiento y el desarrollo de las larvas (Van der Moezel et al., 1987).

Las abejas en su actividad diaria y periódica muestran un alto grado de constancia y son altamente selectivas (Free, 1963) utilizando como fuente de polen sólo algunas de las especies en flor disponibles (Montenegro et al., 1989; Sempe et al., 1989; Montenegro et al., 1990). En cada uno de sus viajes recolectores, visitan mayoritariamente un determinado tipo de flor (Waddington y Holden, 1979), y el 
polen colectado constituye cúmulos coloreados que son transportados a la colmena en canastillos especiales o corbículas ubicadas en el tercer par de patas.

El análisis del polen corbicular realizado utilizando como herramienta su especificidad, permitirá establecer cuales son las especies preferidas y el tiempo en que ellas son utilizadas por las abejas melíferas. Este conocimiento favorecerá al apicultor ya que basado en él, podrá seleccionar mejor el lugar para ubicar las colmenas.

El objetivo de este trabajo fue diagnosticar la flora nativa utilizada por Apis mellifera en un área de la Séptima Región de Chile, que comprende la Reserva Nacional Los Ruiles, zona bioclimática mediterrảnea sub-húmeda, cuantificando la importancia relativa de cada especie colectada, a través de la biomasa de los cúmulos corbiculares. Con los resultados obtenidos a través de estudios similares en otros sitios de la zona mediterránea (Montenegro et al., 1989; Sempe et al., 1989; Montenegro et al., 1990), se podrá establecer una red fenológica nacional y determinar similitudes y diferencias de disponibilidad temporal de las especies en diferentes comunidades vegetacionales, al mismo tiempo que permitirá establecer en gradiente de preferencias específicas, contribuyendo así a una optimización del recurso.

\section{MATERIAL Y MÉTODOS}

\section{Situación.}

El colmenar se encuentra ubicado en un lugar vecino a la Reserva Nacional Los Ruiles a $10 \mathrm{Km}$ de la ciudad de Cauquenes en la Séptima Región de Chile (33 59' LS y $72^{\circ} 22^{\prime} \mathrm{LO}$ ) aproximadamente a $100 \mathrm{msnm}$. El clima corresponde al tipo mediterráneo sub-húmedo con temperaturas máximas promedio de $22^{\circ} \mathrm{C}$ y mínimas promedio de $8^{\circ} \mathrm{C}$ (Di Castri y Hajek, 1976).

La vegetación corresponde predominantemente a un matorral originado por la degradación del bosque nativo, en el cual persisten abundantes especies de las familias Mirtaceae, Ericaceae y Fagaceae. La zona de bosque aledaña está dominada por especies del género Nothofagus, que coexisten con especies como: Aextoxicon punctatum, Amomyrtus luma, Luma apiculata, Drimys winteri, Crinodendron patagua, Gevuina avellana y Laurelia sempervirens. El estrato herbáceo en las áreas de matorral presenta abundancia de geófitas entre las cuales se encuentra: Brodiaea porrifolia, Pasithaea coerulea, terófitas como varias especies de Gramíneas, especies del género Erodium e hierbas perennes como Hypochaeris radicata.

\section{Muestreo de polen corbicular.}

El muestreo de especies en flor se hizo una vez al mes, desde septiembre de 1988 a marzo de 1989. Con las muestras se confeccionó un herbario del lugar y se hizo una colección de referencia de preparaciones de polen para microscopía óptica y electrónica de barrido en la que se incluyó también las dimensiones de cada tipo polínico y sus principales características morfológicas.

Mensualmente, coincidiendo con el muestreo de la zona circundante, se obtuvo muestras de cúmulos corbiculares de polen, mediante trampas especiales que se ubicaron a la entrada de las colmenas, desde las 9:30 hrs. y hasta las 17:30 hrs. 


\section{Identificación de especies vegetales en el polen corbicular.}

La muestra de polen corbicular se separó tomando como referencia el color que presentaban los cúmulos, ya que en su mayoría son monoespecíficos (Montenegro et al., datos no publicados). Estos colores se cotejaron con una tabla internacional de colores (Pantone) con el fin de proceder a caracterizarlos y darles el número correspondiente al color.

Con este material se confeccionaron preparaciones tanto para microscopía óptica como para microscopía electrónica de barrido (Fig. 1), las que luego fueron identificadas con ayuda de la colección de referencia y de bibliografía pertinente (Heusser, 1971; Erdtman, 1986).

Para la microscopía óptica se utilizó el método Gómez-Avila que consiste en colocar un trozo de cúmulo.polínico sobre un portaobjetos y luego disolver con alcohol etílico de $50^{\circ}$, dejar secar y teñir con una gota de Calberla, nuevamente secar y cubrir con Entellan.

Para la obtención de preparaciones para microscopía electrónica de barrido, se utilizó el método descrito en Montenegro et al., 1989.

\section{Cuantificación del polen corbicular.}

Los cúmulos polínicos identificados y separados por especies fueron secados en estufa a $50^{\circ} \mathrm{C}$ durante 48 horas para determinar a través de la biomasa la importancia relativa con la cual contribuye cada especie en el total de la muestra de polen colectado en la trampa.

\section{RESULTADOS Y DISCUSIÓN}

Las observaciones en el sitio de estudio, permitieron determinar un total de 141 especies presentes, cuya floración se distribuye a lo largo de los siete meses analizados. El número de especies que florece mensualmente y, por lo tanto, que están disponibles para las abejas, se muestra en la Fig. 2, diferenciándose de este total, las especies utilizadas como fuente de polen por Apis mellifera. Se puede apreciar que, aunque la disponibilidad de especies aumenta en forma significativa en algunos meses del año, la visita de la abejas se mantiene constante a lo largo de los meses, utilizando éstas entre el 10 y el $20 \%$ del total de especies disponibles. La excepción se observa en el mes de Marzo en que, el uso de especies es de alrededor del 6\%, debido, posiblemente, a que por disminución de la temperatura ambiental, la actividad de las abejas también disminuye.

Para las especies preferidas por Apis mellifera se confeccionó un calendario fenológico en el cual se incluye el calendario de utilización (Fig. 3). Se puede observar que cuando comienzan a florecer especies más apetecidas por las abejas, éstas abandonan las que estaban utilizando y sólo en algunos casos vuelven a utilizar las previamente visitadas. Ejemplo de esto último se observa en especies como Azara serrata, Laurelia sempervirens y Berberis darwini, resultando ser Luma apiculata la especie preferida durante 5 meses, correspondiendo al período más largo del muestreo. Esto podría deberse a la gran cantidad de flores que esta especie forma y además a que las flores presentan numerosos estambres fácilmente accesibles asegurando así una significativa recompensa para la abejas (Devesa, 1989). 


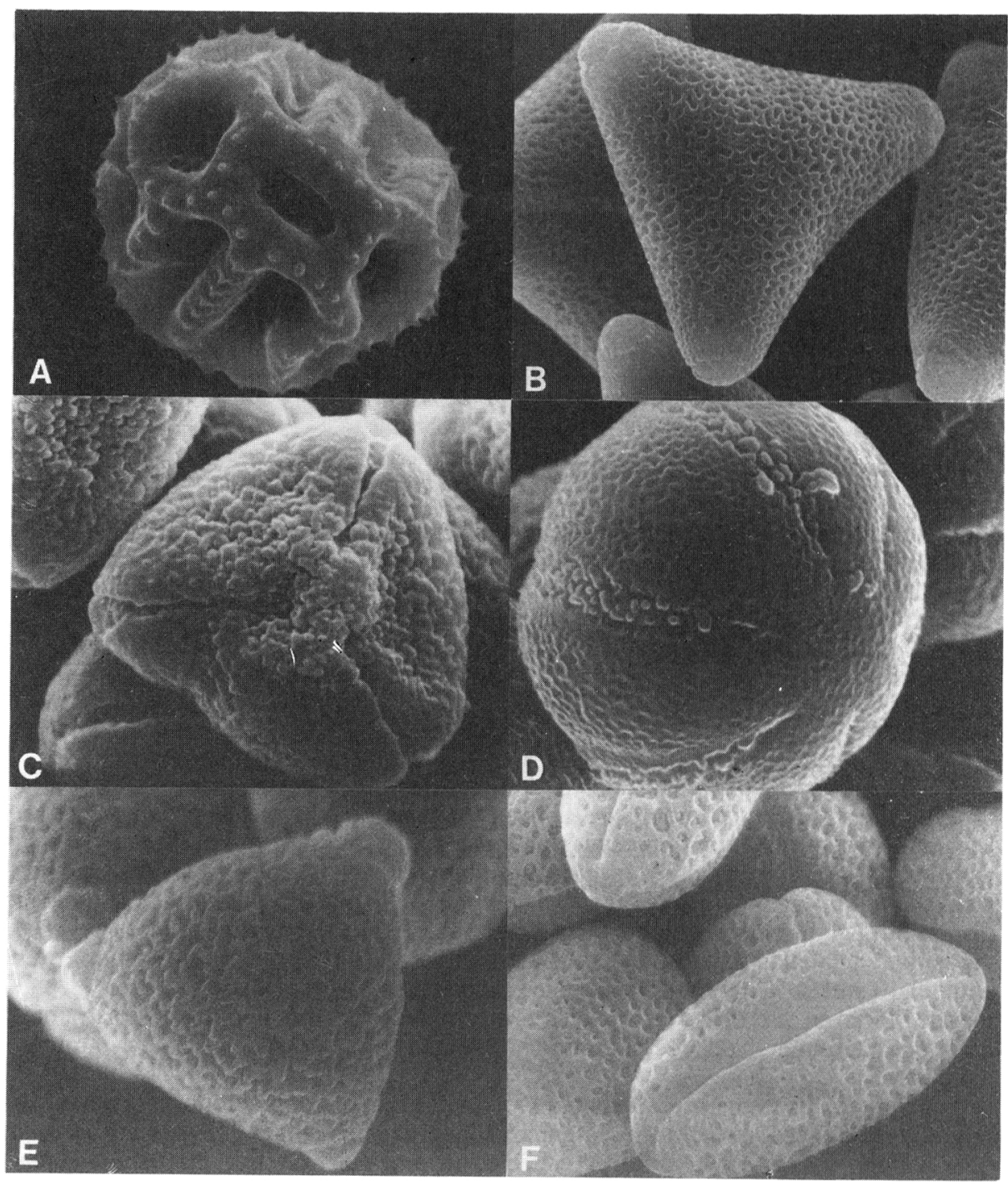

Figura 1. Pólenes fotografiados al Microscopio Electrónico de Barrido. A: Hypochaeris radicata 200x. B: Gevuina avellana 200x. C: Luma apiculata 4500x. D: Mentha pulegium 4500x. E: Lomatia hirsuta 3000 x. F: Azara integrifolia 4500x. 


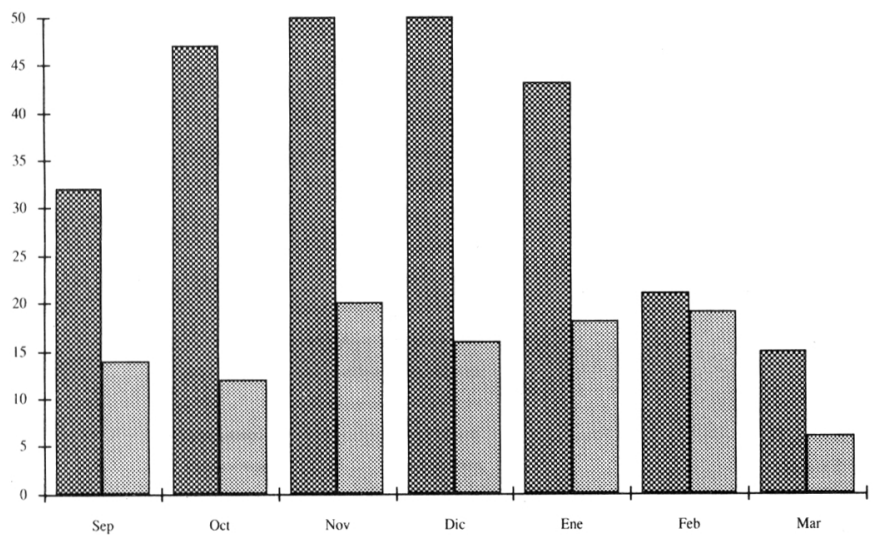

Figura 2. Número de especies en flor durante el período muestreado. Las barras oscuras muestran el total de las especies disponibles y las barras claras el total de las especies utilizadas por las abejas como fuente de polen.

Apis mellifera muestra una selectividad en la utilización de polen y esta selectividad es también cuantitativa, de tal manera que hay especies que son utilizadas significativamente más que otras.

La tabla 1 resume para cada especie visitada por las abejas la cantidad de polen corbicular expresado en términos de biomasa. La cuantificación de las especies presentes en el polen corbicular permitió determinar la frecuencia relativa de cada una de ellas diagnosticándose aquellas de mayor importancia.

Destaca de estos resultados la utilización que hace del polen de Rubus ulmifolius (zarzamora), ya que durante el mes de diciembre es la especie que muestra gran predominio sobre las demás. Resultados obtenidos en estudios similares realizados en sitios cercanos a Talca, Chile (Iturriaga et al., 1991), muestran valores semejantes. Otras especies que contribuyen con significativamente mayor cantidad de polen $(\mathrm{P}<0.05)$ a la muestra son: Amomyrtus luma, Baccharis pingraea y Gevuina avellana todas estas correspondientes a especies nativas. Además de Rubus ulmifolius, especies introducidas como Teline monspessulana, Malus pumila, Hypochoeris radicata, Mentha pulegium, son también importantes en el acopio de polen.

Los resultados mostraron también que aproximadamente el $55 \%$ de las especies (Tabla 1), corresponde a especies nativas y el $45 \%$ a introducidas, observándose la tendencia a utilizar mayor número de especies nativas en la primera mitad de ciclo (septiembre a noviembre) y especies introducidas preferentemente en la segunda mitad (diciembre a marzo).

Dado el creciente aumento de la actividad apícola en la séptima región y a nivel nacional, debido a la introducción de cultivos no tradicionales en el país que requieren de la intervención de abejas como polinizadores, es bueno crear conciencia que el recurso flora nativa presenta una gran potencialidad para mantener apiarios. Por otro lado el conocimiento de las especies nativas que las abejas utilizan como fuente de polen es fundamental para que el agricultor puede maximizar la eficiencia en los procesos de polinización, ubicando las colmenas en lugares donde disminuya la competencia entre cultivos y flora nativa. 


\begin{tabular}{llllllllll}
\hline Especies & Origen & \multicolumn{10}{c}{ Meses } \\
& A & S & O & N & D & E & F & M
\end{tabular}

Drimys winteri

Teline monspessulana

Cydonia oblonga

Azara serrata

Discaria serratifolia

Laurelia sempervirens

Aristotelia chilensis

Amomyrtus luma

Malus pumila

Calceolaria nudicaulis

Berberis darwini

Prunus domestica

Muehlenbeckia hastulata

Digitalis purpurea

Baccharis elaeoides

Luma apiculata

Myrceugenia exsucca

Colletia ulicina

Calceolaria integrifolia

Nothofagus obliqua

Lomatia hirsuta

Hypachoeris radicata

Prunus persica

Maytenus boaria

Carduus pycnocephalus

$\longrightarrow$

Blepharocalyx cruckshanksii $\mathrm{N}$

Brassica rapa

Gochnatia foliolosa

Lomatia dentata

Zea mays

Rubus ulmifolius

Verbascum virgatum

Gevuina avellana

Stachys albicaulis

Cirsium vulgare

Cytisus scoparius

Foeniculum vulgare

Baccharis pingraea

Luma gayana

Sanicula sp.

Adesmia concina

Rosa moschata

Mentha pulegium

Poligonum sp.

Ugni molinae

Acacia caven

Sphacelle chamaedryoides

Prunella vulgaris

Rumex sp.

Raphanus sativus

Sysimbrium officinalis

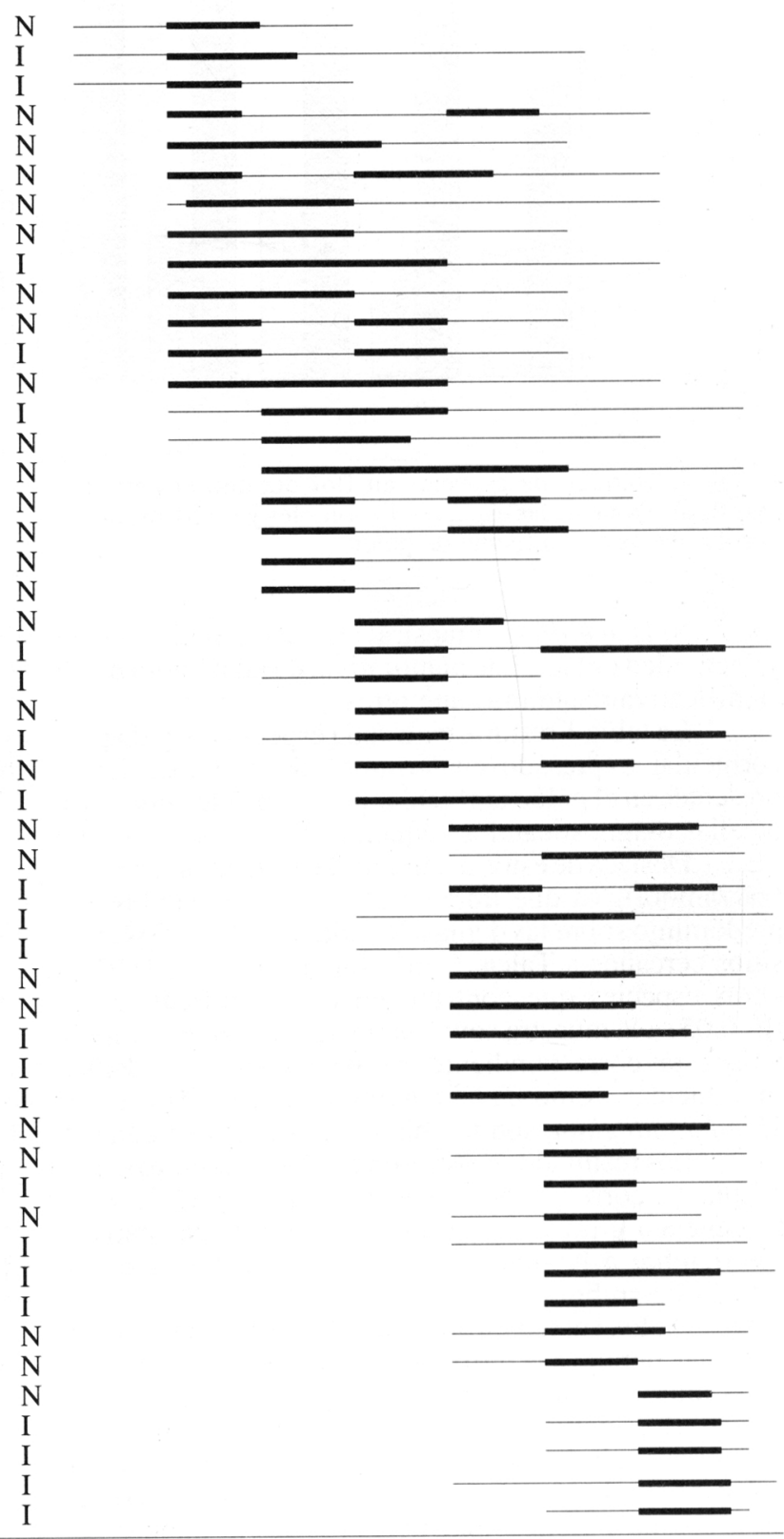

Figura 3. Fenología de la floración de especies utilizadas por Apis mellifera. Trazo fino: período de floración. Trazo grueso: período de utilización. 


\begin{tabular}{|c|c|c|c|c|c|c|c|c|c|}
\hline \multirow[t]{2}{*}{ ESPECIES } & \multicolumn{5}{|c|}{ MESES } & \multicolumn{3}{|r|}{ TOTAL } & \multirow[t]{2}{*}{$\%$} \\
\hline & $S$ & 0 & $\mathrm{~N}$ & $\mathrm{D}$ & $\mathrm{E}$ & $\mathrm{F}$ & M & & \\
\hline Drimys winteri & 72 & - & - & - & - & - & - & 72 & 0,36 \\
\hline Teline monspessulana & 88 & 373 & 342 & - & - & - & - & 803 & 4,12 \\
\hline Cydonia oblonga & 308 & - & - & - & - & - & - & 308 & 1,16 \\
\hline Azara serrata & 168 & - & - & 28 & - & - & - & 196 & 1,00 \\
\hline Discaria serratifolia & 599 & 50 & 85 & - & - & - & - & 737 & 3,77 \\
\hline Laurelia sempervirens & 492 & & 62 & 64 & - & - & - & 618 & 3,17 \\
\hline Aristotelia chilensis & 351 & 163 & 210 & - & - & - & - & 724 & 3,71 \\
\hline Amomyrtus luma & 389 & 512 & 728 & - & - & - & - & 1.629 & 8,35 \\
\hline Malus pumila & 198 & 351 & 252 & 32 & - & - & - & 833 & 4,27 \\
\hline Calceolaria nudicaulis & 98 & 63 & 49 & - & - & - & - & 210 & 1,07 \\
\hline Berberis darwini & 48 & - & 73 & - & - & - & - & 121 & 0,62 \\
\hline Prun & 68 & - & 62 & - & - & - & - & 130 & 0,66 \\
\hline Muehlenbeckia hastulata & 87 & 286 & 157 & 54 & - & - & - & 584 & 2,99 \\
\hline Digitalis purpurea & - & 97 & 58 & 62 & - & - & - & 217 & 1,11 \\
\hline aris elaeoides & - & 101 & 77 & - & - & - & - & 178 & 0,91 \\
\hline Luma apiculata & - & 367 & 205 & 172 & 102 & 111 & - & 957 & 4,91 \\
\hline Myrceugenia exsucca & - & 267 & - & 64 & - & - & - & 331 & 1,69 \\
\hline Collet & - & 218 & - & 135 & 135 & 72 & - & 425 & 2,18 \\
\hline ia integrifolia & - & 35 & - & - & - & - & - & 35 & 0,17 \\
\hline Nothofagus obliqua & - & 72 & - & - & - & - & - & 72 & 0,36 \\
\hline hirsuta & - & - & 111 & 74 & - & - & - & 185 & 0,94 \\
\hline adicata & - & - & 157 & - & 242 & 427 & 232 & 1.058 & 5,42 \\
\hline Prun & - & - & 62 & - & - & - & - & 62 & 0,31 \\
\hline Mayt & - & - & 210 & - & - & - & - & 210 & 1,07 \\
\hline enhallus & - & - & 71 & - & 74 & 219 & 187 & 461 & 2,36 \\
\hline Blapharocalyx cruchshanksii & - & - & 69 & - & 128 & - & - & 197 & 1,01 \\
\hline$a$ & - & - & 48 & 69 & 94 & 84 & - & 295 & 1,51 \\
\hline tia foliolosa & - & - & - & 102 & 322 & 86 & 106 & 634 & 3,25 \\
\hline a dentata & - & - & - & & 71 & 153 & - & 225 & 1,15 \\
\hline Zea & - & - & - & 74 & - & 247 & - & 321 & 1,64 \\
\hline Rubl & - & - & - & 1.678 & 351 & 70 & - & 2.099 & 10,76 \\
\hline Verb & - & - & - & 55 & - & - & - & 55 & 0,28 \\
\hline Gev & - & - & - & 154 & 318 & 361 & - & 833 & 4,27 \\
\hline & - & - & - & 42 & 78 & 62 & 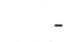 & 182 & 0,93 \\
\hline Cirs & - & - & - & 38 & 241 & 251 & 218 & 748 & 3,87 \\
\hline & - & - & - & 54 & 64 & - & - & 118 & 0,60 \\
\hline & - & - & - & 40 & 43 & - & - & 83 & 0,42 \\
\hline $\mathrm{Bacc}$ & - & - & - & - & 132 & 368 & 610 & 1.110 & 5,69 \\
\hline & - & - & - & - & 71 & - & - & 71 & 0,36 \\
\hline & - & - & - & - & 58 & - & - & 58 & 0,29 \\
\hline ncina & - & - & - & - & 101 & - & - & 101 & 0,51 \\
\hline & - & - & - & - & 112 & - & - & 112 & 0,57 \\
\hline & - & - & - & - & 115 & 92 & 171 & 378 & 1,93 \\
\hline Poligonum sp. & - & - & - & - & 101 & - & - & 101 & 0,51 \\
\hline Ugni molinae & - & - & - & - & 60 & 139 & - & 199 & 1,02 \\
\hline & - & - & - & - & 51 & - & - & 51 & 0,26 \\
\hline Sphacelle chamaedryoides & - & - & - & - & - & 97 & - & 97 & 0,49 \\
\hline Prunella vulgaris & - & - & - & - & - & 103 & - & 103 & 0,52 \\
\hline & - & - & - & - & - & 66 & - & 65 & 0,33 \\
\hline Raphanus sativus & - & - & - & - & - & 42 & 38 & 80 & 0,41 \\
\hline Sysimbrium officinalis & - & - & - & - & - & 42 & - & 42 & 0,21 \\
\hline
\end{tabular}

Tabla 1. Cantidad de polen corbicular expresado en miligramos de biomas. Los valores corresponden a un total de 3 gramos por muestra de polen recolectado en las trampas. 
AGRADECIMIENTOS. Nuestros especiales agradecimiento al Sr. Luis Mouchet que permitió muestrear sus colmenas y recorrer su predio para analizar la vegetación circundante. Este trabajo se realizó con financiamiento del Fondo Nacional de Ciencias a través del Proyecto FONDECYT 747/91.

\section{BIBLIOGRAFÍA}

DEVESA, J.A. -1989- La función polinizadora de la abeja en los frutales de hueso. Vida Apícola, 37: 52-55.

DI CASTRI, F. y E.R. HAJEK -1976-Bioclimatología de Chile. Vicerrectoría Académica de la Universidad Católica de Chile. 129 pp.

ERDTMAN, G. -1986- Polen morphology and plant taxonomy. Angiosperms. An introduction to palinology. E.J. Brill. Leiden. The Netherlands $553 \mathrm{pp}$.

FREE, J.B. -1963- The flower constancy of honey bees. J. Anim. Ecol., 32:119-131.

ITURRIAGA, L., G. AVILA, M. GOMEZ, S. TEILLIER Y G. MONTENEGRO-1991-Especies vegetales utilizadas como fuente de polen por Apis mellifera en la región mediterránea subhúmeda de Chile. Simiente (en prensa).

HEUSSER, J. -1971- Polen and spores of Chile. Tucson. The University of Arizona Press.

MONTENEGRO, G., M. SCHUCK, A.M. MUJICA y S. TEILLIER -1989- Flora utilizada por abejas melíferas (Apis mellifera) como fuente de polen en Paine, Región Metropolitana, Chile. Ciencia e Inv. Agr., 16(1-2): 47-54.

MONTENEGRO, G. G. AVILA, E. CARDALDA, M. COTTENIE, R. GINOCCHIO, M. GOMEZ, L.ITURRIAGA, L. GONZALEZ, A.M. MUJICA. V.POBLETE, G. RIZZARDINI, M. SCHUCK, A. SILVA, C. SILVA, J. SEMPE, S. TEILLIER, Y D. VARELA -1990Implementación de una red fenológica de especies melíferas. En Actas: II Encuentro Nacional de Ciencia y Tecnología Apícola. L. Alda, R. Rebolledo y D. Ríos (eds). Universidad de La Frontera. pp. 149-176.

SEMPE, J., C. RAMIREZ Y G. MONTENEGRO -1989- Flora utilizada como fuente de polen por Apis mellifera en la provincia de Valdivia: análisis cuantitativo del polen corbicular. Ciencia e Inv. Agr., 16(1-2): 55-65.

VAN DER MOEZEL, P.G., J.C. DELFS, J.S. PATE, W.A. LORENAGAN \& D.T. BELL -1987Pollen selection by Honeybees in shrublands of the northern sandplains of western Australia. Journal of Apicultural Research, 26(4): 224-232.

WADDINGTON, K.D. \& L.R. HOLDEN -1979- Optimal foraging: on flower selection by bees. Am. Nat., 114:179-189.

(Aceptado para su publicación en junio de 1991)

Dirección de los autores: Departamento de Ecología. Facultad de Ciencias Biológicas. Pontificia Universidad Católica de Chile. Santiago. Chile. 